\title{
Falkner-Skan Flow of a Maxwell Fluid with Heat Transfer and Magnetic Field
}

\author{
M. Qasim ${ }^{1}$ and S. Noreen ${ }^{2}$ \\ ${ }^{1}$ Department of Mathematics, COMSATS Institute of Information Technology, Park Road, Chak Shahzad, Islamabad 44000, Pakistan \\ ${ }^{2}$ Department of Mathematics, COMSATS Institute of Information Technology, Attock 43600, Pakistan
}

Correspondence should be addressed to M. Qasim; mq_qau@yahoo.com

Received 11 February 2013; Accepted 19 May 2013

Academic Editor: George S. Dulikravich

Copyright () 2013 M. Qasim and S. Noreen. This is an open access article distributed under the Creative Commons Attribution License, which permits unrestricted use, distribution, and reproduction in any medium, provided the original work is properly cited.

\begin{abstract}
This investigation deals with the Falkner-Skan flow of a Maxwell fluid in the presence of nonuniform applied magnetic field with heat transfer. Governing problems of flow and heat transfer are solved analytically by employing the homotopy analysis method (HAM). Effects of the involved parameters, namely, the Deborah number, Hartman number, and the Prandtl number, are examined carefully. A comparative study is made with the known numerical solution in a limiting sense and an excellent agreement is noted.
\end{abstract}

\section{Introduction}

The Falkner-Skan problem under various aspects has attracted the attention of several researchers [1]. This problem under various aspects has been discussed extensively for viscous fluid. The interested readers may consult the studies in [2-11] for detailed information in viscous fluids. There are several materials which do not obey the Newton's law of viscosity, for example, biological products like blood and vaccines, foodstuffs like honey, ketchup, butter, and mayonnaise, certain paints, cosmetic products, pharmaceutical chemicals and so forth. These fluids are characterized as the non-Newtonian fluids. Investigation of such fluids is very useful in industrial, engineering, and biological applications. However, such fluids cannot be studied by employing a single constitutive relationship. This is due to diverse properties of non-Newtonian fluids in nature. These non-Newtonian fluid models are discussed in view of three main categories, namely, the differential, the rate, and the integral types. The simplest subclass of rate type fluids is called Maxwell. The Maxwell fluid allows for the relaxation effects which cannot be predicted in differential type fluids, namely, second, third, and fourth grades. Recently, there has been an increasing interest in the theory of rate type fluids and, in particular, a Maxwell fluid model has been accorded much attention.
The Falkner-Skan wedge flow of a non-Newtonian fluid was firstly investigated by Rajagopal et al. [12]. Massoudi and Ramezan [13] discussed the effect of injection or suction on the Falkner-Skan flows of second grade fluids. The Falkner-Skan wedge flow of power-law fluids embedded in a porous medium is investigated by Kim [14]. Olagunju [15] studied this flow problem for viscoelastic fluid. In [10-15], the attention has been given to the differential type fluids. To the best of our knowledge, no one investigated the Falkner-Skan flow problem for rate type fluids.

In [10], Yao has examined the Falkner-Skan wedge flow. $\mathrm{He}$ established series solution for the velocity and temperature by using homotopy analysis method [16-25]. The purpose of the present contribution is to extend the flow analysis of study [10] in two directions. The first generalization is concerned with the consideration of electrically conducting fluid. Such analysis has inserted in power generators the cooling of reactors, MHD accelerators, the design of heat exchanges and electrostatic filters. Secondly, we consider the Maxwell fluid instead of viscous fluid. The rest of the paper is arranged as follows. The description of the problem is presented in Section 2. Section 3 develops the homotopy solutions for velocity and temperature. Convergence of the derived solution is examined in Section 4. Further, the variations of embedded parameters have been discussed in this section. 


\section{Problem Development}

We study the steady two-dimensional flow of a Maxwell fluid in the presence of a magnetic field. The magnetic Reynolds number is small so that induced magnetic field is neglected. The stream velocity $U(x)$ varies according to $x^{n}$. The constant temperature of surface and free stream is $T_{w}$ and $T_{\infty}$, respectively. The boundary layer equations for the considered problem are

$$
\begin{gathered}
\frac{\partial u}{\partial x}+\frac{\partial v}{\partial y}=0 \\
u \frac{\partial u}{\partial x}+v \frac{\partial u}{\partial y}+\lambda\left(u^{2} \frac{\partial^{2} u}{\partial x^{2}}+v^{2} \frac{\partial^{2} u}{\partial y^{2}}+2 u v \frac{\partial^{2} u}{\partial x \partial y}\right) \\
=v \frac{\partial^{2} u}{\partial y^{2}}+U \frac{\partial U}{\partial x}+\lambda U^{2} \frac{\partial^{2} U}{\partial x^{2}}-\frac{\sigma B^{2}}{\rho}\left(u-U+\lambda v \frac{\partial u}{\partial y}\right) \\
u \frac{\partial T}{\partial x}+v \frac{\partial T}{\partial y}=\frac{k}{\rho c_{p}} \frac{\partial^{2} T}{\partial y^{2}}
\end{gathered}
$$

where $u$ and $v$ are the velocity components in the $x$ - and $y$-directions, respectively, $v$ is kinematic viscosity, $\sigma$ is the electrical conductivity, $k$ is the thermal conductivity, $\rho$ is the fluid density, $T$ is the fluid temperature, $\lambda$ is relaxation time, $B$ is the magnetic field, and $c_{p}$ is specific heat. lows:

The relevant boundary conditions are prescribed as fol-

$$
\begin{aligned}
& u=0, \quad v=0, \quad T=T_{w} \quad \text { at } y=0, \\
& u \longrightarrow U(x), \quad T \longrightarrow T_{\infty} \quad \text { as } y \longrightarrow \infty,
\end{aligned}
$$

with [1]

$$
U(x)=a x^{n}
$$

and $[7,8]$

$$
B(x)=B_{0} x^{(n-1) / 2}
$$

Putting

$$
\begin{gathered}
\psi=\sqrt{\frac{2}{n+1}} \sqrt{v x U} f(\eta), \\
\eta=\sqrt{\frac{n+1}{2}} \sqrt{\frac{U}{x \nu}} y, \quad u=U f^{\prime}(\eta), \\
v=-\sqrt{\frac{n+1}{2}} \sqrt{\frac{U}{x \nu}}\left[f(\eta)+\frac{n-1}{n+1} \eta f^{\prime}(\eta)\right], \\
\theta(\eta)=\frac{T_{w}-T}{T_{w}-T_{\infty}}, \\
u=\frac{\partial \psi}{\partial y}, \quad v=-\frac{\partial \psi}{\partial x},
\end{gathered}
$$

(1) is satisfied identically and (2)-(4) give

$$
\begin{aligned}
& f^{\prime \prime \prime}+f f^{\prime \prime}+\frac{2 n}{n+1}\left(1-f^{\prime 2}\right) \\
& -M^{2}\left(f^{\prime}-1\right)+\left(M^{2} \beta+1\right) f f^{\prime \prime} \\
& +\beta\left(2 n \frac{n-1}{n+1}\left(1-f^{\prime 3}\right)+(3 n-1) f f^{\prime} f^{\prime \prime}\right. \\
& \left.-\frac{n+1}{2} f^{2} f^{\prime \prime \prime}+\frac{n-1}{2} \eta f^{\prime 2} f^{\prime \prime}\right)=0, \\
& \theta^{\prime \prime}+\operatorname{Pr} f \theta^{\prime}=0, \\
& f^{\prime}(0)=0, \quad \theta(0)=0, \\
& f^{\prime}(\infty)=1, \quad \theta(\infty)=1 .
\end{aligned}
$$

Here $\psi$ is the stream function, prime denotes the differentiation with respect to $\eta, \gamma$ is the local Deborah number, $n$ is the constant parameter, $M$ is Hartmann number, and $\operatorname{Pr}$ is the Prandtl number. The values of $\beta, M$, and Pr are

$$
\beta=\frac{\lambda U}{x}, \quad M=\frac{2 \sigma B_{0}^{2}}{\rho a(n+1)}, \quad \operatorname{Pr}=\frac{\mu c_{p}}{k} .
$$

The local Nusselt number $N u_{x}$ and heat transfer from the plate $q_{w}$ are given by

$$
N u_{x}=\frac{x q_{w}}{k\left(T_{w}-T_{\infty}\right)}, \quad q_{w}=-k\left(\frac{\partial T}{\partial y}\right)_{y=0} .
$$

Invoking (7) one obtains

$$
\operatorname{Re}_{x}^{-1 / 2}\left(\frac{n+1}{2}\right) N u_{x}=-\theta^{\prime}(0)
$$

in which the local Reynolds number $\operatorname{Re}_{x}=U x / \nu$. For $M=$ $0=\beta$, from (9), we have

$$
f^{\prime \prime \prime}+f f^{\prime \prime}+\gamma\left(1-f^{\prime 2}\right)=0
$$

where $\gamma=2 n /(n+1)$.

\section{Homotopy Analysis Solutions}

For an interest in homotopy solutions, we express $f(\eta)$ and $\theta(\eta)$ by a set of base functions

$$
\begin{gathered}
\left\{\eta^{m} \exp (-2 n \eta), m, n \geq 0\right\}, \\
\left\{\eta^{m} \exp (-n \eta), m, n \geq 0\right\},
\end{gathered}
$$

as follows:

$$
\begin{aligned}
& f(\eta)=\sum_{m=0}^{\infty} \sum_{n=0}^{\infty} a_{m, n} \eta^{m} \exp (-2 n \eta), \\
& \theta(\eta)=\sum_{m=0}^{\infty} \sum_{n=0}^{\infty} b_{m, n} \eta^{m} \exp (-2 n \eta)
\end{aligned}
$$


where $a_{m, n}$ and $b_{m, n}$ are the coefficients. The initial approximations of $f(\eta)$ and $\theta(\eta)$ and the auxiliary linear operators $\mathscr{L}_{f}$ and $\mathscr{L}_{\theta}$ are

$$
\begin{gathered}
f_{0}(\eta)=\eta-\frac{1-\exp (-2 \eta)}{2}, \quad \theta_{0}(\eta)=1-\exp (-\eta) \\
\mathscr{L}_{f}(f)=\frac{d^{3} f}{d \eta^{3}}-4 \frac{d f}{d \eta}, \quad \mathscr{L}_{\theta}(\theta)=\frac{d^{2} \theta}{d \eta^{2}}+\frac{d \theta}{d \eta} \\
\mathscr{L}_{f}\left[C_{1}+C_{2} \exp (2 \eta)+C_{3} \exp (-2 \eta)\right]=0 \\
\mathscr{L}_{\theta}\left[C_{4} \exp (\eta)+C_{5} \exp (-\eta)\right]=0
\end{gathered}
$$

in which $C_{i}(i=1-5)$ are the arbitrary constants. If $p \in$ $[0,1]$ is the embedding parameter and $\hbar_{f}, \hbar_{\theta}$ and $\hbar_{\phi}$, are the nonzero auxiliary parameters, then the problems at zeroth order give

$$
\begin{aligned}
& (1-p) \mathscr{L}_{f}\left[\widehat{f}(\eta ; p)-f_{0}(\eta)\right]=p \hbar_{f} \mathcal{N}_{f}[\widehat{f}(\eta ; p)], \\
& (1-p) \mathscr{L}_{\theta}\left[\hat{\theta}(\eta ; p)-\theta_{0}(\eta)\right]=p \hbar_{\theta} \mathscr{N}_{\theta}[\hat{\theta}(\eta ; p), \widehat{f}(\eta ; p)], \\
& \widehat{f}(0 ; p)=0, \quad \hat{f}^{\prime}(0 ; p)=0, \quad \hat{f}^{\prime}(\infty ; p)=1, \\
& \widehat{\theta}(0 ; p)=0, \quad \widehat{\theta}(\infty ; p)=1, \\
& \mathscr{N}_{f}[\widehat{f}(\eta ; p)] \\
& =\frac{\partial^{3} \widehat{f}(\eta ; p)}{\partial \eta^{3}}+\widehat{f}(\eta ; p) \frac{\partial^{2} \widehat{f}(\eta ; p)}{\partial \eta^{2}} \\
& +\frac{2 n}{n+1}\left[1-\left(\frac{\partial \widehat{f}(\eta, p)}{\partial \eta}\right)^{2}\right] \\
& -M^{2}\left(\frac{\partial \hat{f}(\eta, p)}{\partial \eta}-1\right) \\
& +\left(M^{2} \beta+1\right) \frac{\partial \hat{f}(\eta, p)}{\partial \eta} \frac{\partial^{2} \widehat{f}(\eta, p)}{\partial \eta^{2}} \\
& +\beta\left[2 n \frac{n-1}{n+1}\left[1-\left(\frac{\partial \widehat{f}(\eta, p)}{\partial \eta}\right)^{3}\right]\right. \\
& +(3 n-1) \widehat{f}(\eta ; p) \frac{\partial \widehat{f}(\eta, p)}{\partial \eta} \frac{\partial^{2} \widehat{f}(\eta ; p)}{\partial \eta^{2}}
\end{aligned}
$$

$$
\mathcal{N}_{\theta}[\widehat{\theta}(\eta ; p), \widehat{f}(\eta ; p)]=\frac{\partial^{2} \widehat{\theta}(\eta, p)}{\partial \eta^{2}}+\operatorname{Pr} \widehat{f}(\eta ; p) \frac{\partial \widehat{\theta}(\eta ; p)}{\partial \eta} .
$$

For $p=0$ and $p=1$, we have

$$
\begin{array}{ll}
\hat{f}(\eta ; 0)=f_{0}(\eta), & \widehat{f}(\eta ; 1)=f(\eta), \\
\hat{\theta}(\eta ; 0)=\theta_{0}(\eta), & \widehat{\theta}(\eta ; 1)=\theta(\eta) .
\end{array}
$$

When $p$ increases from 0 to $1, \widehat{f}(\eta ; p)$ and $\widehat{\theta}(\eta ; p)$ vary from $f_{0}(\eta)$ and $\theta_{0}(\eta)$ to the exact solutions $f(\eta)$ and $\theta(\eta)$. In view of Taylors theorem and (20) and (21), one arrives at

$$
\begin{gathered}
\widehat{f}(\eta ; p)=f_{0}(\eta)+\sum_{m=1}^{\infty} f_{m}(\eta) p^{m}, \\
\hat{\theta}(\eta ; p)=\theta_{0}(\eta)+\sum_{m=1}^{\infty} \theta_{m}(\eta) p^{m}, \\
f_{m}(\eta)=\left.\frac{1}{m !} \frac{\partial^{m} \widehat{f}(\eta ; p)}{\partial p^{m}}\right|_{p=0}, \\
\theta_{m}(\eta)=\left.\frac{1}{m !} \frac{\partial^{m} \hat{\theta}(\eta ; p)}{\partial p^{m}}\right|_{p=0} .
\end{gathered}
$$

The auxiliary parameters are so properly chosen that the series (28) and (29) converge at $p=1$, and hence

$$
\begin{aligned}
& f(\eta)=f_{0}(\eta)+\sum_{m=1}^{\infty} f_{m}(\eta), \\
& \theta(\eta)=\theta_{0}(\eta)+\sum_{m=1}^{\infty} \theta_{m}(\eta) .
\end{aligned}
$$

The problems at $m$ th-order deformation satisfy the following equations and boundary conditions:

$$
\begin{gathered}
\mathscr{L}_{f}\left[f_{m}(\eta)-\chi_{m} f_{m-1}(\eta)\right]=\hbar_{f} \mathscr{R}_{m}^{f}(\eta), \\
\mathscr{L}_{\theta}\left[\theta_{m}(\eta)-\chi_{m} \theta_{m-1}(\eta)\right]=\hbar_{\theta} \mathscr{R}_{m}^{\theta}(\eta), \\
f_{m}(0)=f_{m}^{\prime}(0)=f_{m}^{\prime}(\infty)=f_{m}^{\prime \prime}(\infty)=0, \\
\theta_{m}(0)=\theta_{m}(\infty)=0,
\end{gathered}
$$




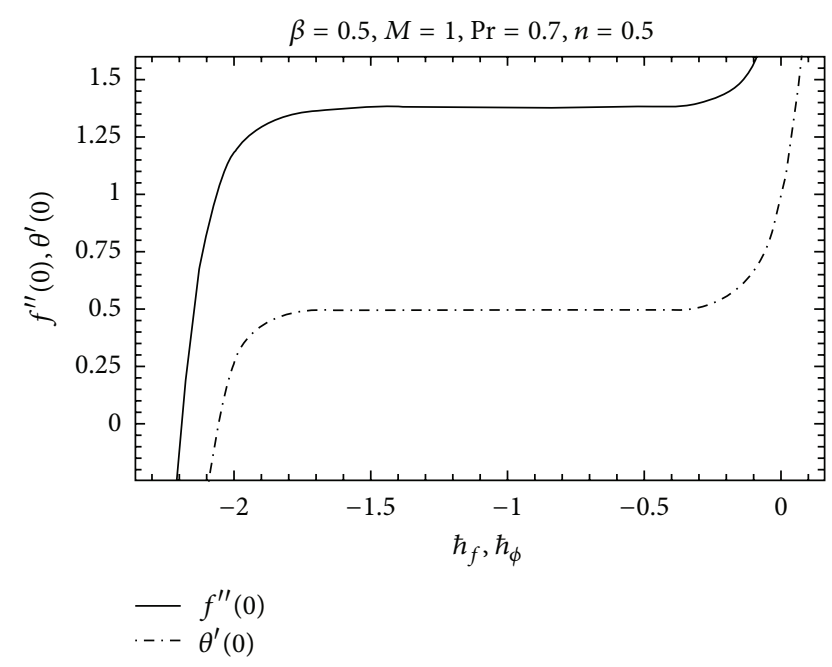

FIGURE 1: $\hbar$ curves for 15 th-order approximations.

$$
\begin{aligned}
& \mathscr{R}_{m}^{f}(\eta)= f_{m-1}^{\prime \prime \prime}(\eta) \\
&+\sum_{k=0}^{m-1}\left[f_{m-1-k} f_{k}^{\prime \prime}+\frac{2 n}{n+1}\left(1-f_{m-1-k}^{\prime} f_{k}^{\prime}\right)\right. \\
&\left.+\left(M^{2} \beta+1\right) f_{m-1-k}^{\prime} f_{k}^{\prime \prime}\right] \\
&+\beta \sum_{k=0}^{m-1}\left[\begin{array}{l}
2 n \frac{n-1}{n+1}\left(1-f_{m-1-k}^{\prime} \sum_{l=0}^{k} f_{k-l}^{\prime} f_{l}^{\prime \prime}\right) \\
+
\end{array}\right. \\
& \quad-\frac{(3 n-1) f_{m-1-k} \sum_{l=0}^{k} f_{k-l}^{\prime} f_{l}^{\prime \prime}}{2} f_{m-1-k}^{\prime \prime} \sum_{l=0}^{k} f_{k-l}^{\prime} f_{l}^{\prime \prime \prime} \\
&\left.+\frac{n-1}{2} f_{m-1-k}^{\prime} \sum_{l=0}^{k} f_{k-l}^{\prime} f_{l}^{\prime \prime}\right] \\
& \chi_{m}^{\theta}(\eta)= \\
& \theta_{m-1}^{\prime \prime}+\operatorname{Pr} \sum_{k=0}^{m-1}\left[\theta_{m-1-k}^{\prime} f_{k}\right], \\
& m \leq 1 \\
& m>1 .
\end{aligned}
$$

\section{Convergence of the Homotopy Solutions}

Obviously the auxiliary parameters $\hbar_{f}$ and $\hbar_{\theta}$ in the series solutions (31) and (32) have a definite role in adjusting and controlling the convergence. For the admissible values of $\hbar_{f}$ and $\hbar_{\theta}$, the $\hbar_{f}$ and $\hbar_{\theta}$ curves are portrayed for 15 thorder of approximations. It is noticed that the ranges for the admissible values of $\hbar_{f}$ and $\hbar_{\theta}$ are $-1.5 \leq \hbar_{f} \leq-0.2$ and $-1.2 \leq \hbar_{\theta} \leq-0.6$ (Figure 1). Moreover, the series given by (31)

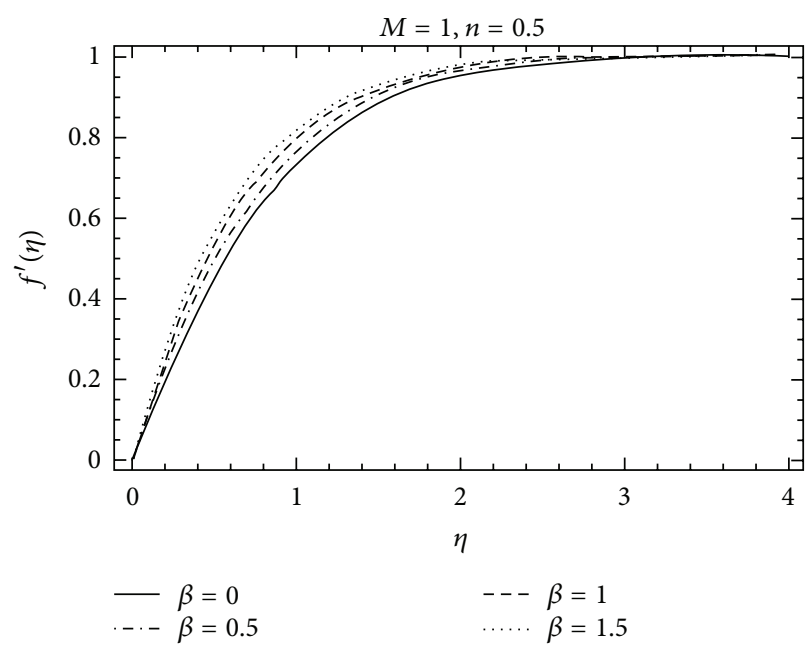

Figure 2: Influence of $\beta$ on $f^{\prime}$.

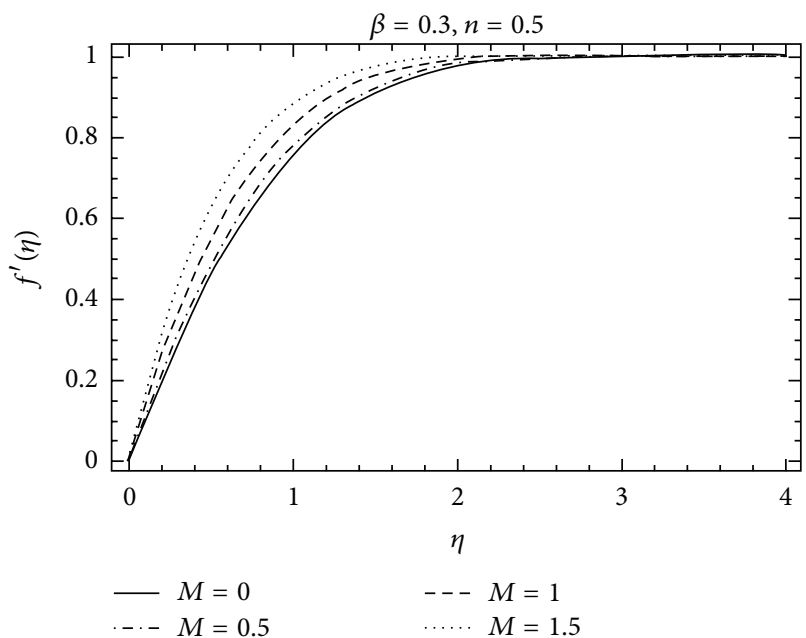

Figure 3: Influence of $M$ on $f^{\prime}$.

and (32) converge in the whole region of $\eta$ when $\hbar_{f}=-0.5$ and $\hbar_{\theta}=-0.8$.

\section{Results and Discussion}

The purpose of this section is to investigate the variations of parameters including Deborah number $\gamma$, the constant $n$, Hartman number $M$, and the Prandtl number $\operatorname{Pr}$ on the velocity $f^{\prime}$ and the temperature $\theta$. The variation of the Deborah number $\beta$, Hartman number $M$, and the parameter $n$ on the velocity $f^{\prime}$ can be seen in Figures $2-5$. It is found from Figure 2 that the boundary layer thickness decreases with an increase in $\gamma$. Figure 3 is plotted for the effects of Hartman number $M$ on the velocity profile $f^{\prime}$. The effects of $M$ on $f^{\prime}$ are qualitatively similar to those of $\gamma$. Figure 4 depicts the velocity field for different values of parameter $n$. Clearly $f^{\prime}$ is an increasing function of $n$ while the boundary layer thickness decreases. Figure 5 shows the effects of Prandtl number Pr on the temperature profile $\theta$. The thermal 
TABLE 1: Comparison of the results $\theta^{\prime}(\eta)$ when $\beta=0=M$.

\begin{tabular}{lccccrr}
\hline$\eta$ & \multicolumn{2}{c}{$f(\eta)$} & & $f^{\prime}(\eta)$ & & $f^{\prime \prime}(\eta)$ \\
\hline 0.0 & HAM & HAM & HAM & 0.469600 \\
0.5 & 0.000000 & 0.00000 & 0.000000 & 0.000000 & 0.46960 \\
1.0 & 0.058643 & 0.05864 & 0.234228 & 0.23423 & 0.465038 & 0.434379 \\
2.0 & 0.232990 & 0.23299 & 0.460633 & 0.46063 & 0.255669 & 0.43438 \\
3.0 & 0.886821 & 0.86680 & 0.816695 & 0.96905 & 0.067713 & 0.06771 \\
4.0 & 1.795568 & 1.79557 & 0.969092 & 0.99777 & 0.006875 & 0.00687 \\
5.0 & 2.783886 & 2.78388 & 0.997770 & 0.99994 & 0.00258 & 0.00026 \\
\hline
\end{tabular}

TABLE 2: Comparison of the results when $M=0=\beta$.

\begin{tabular}{|c|c|c|c|c|c|c|}
\hline \multirow{2}{*}{$\operatorname{Pr}$} & \multicolumn{2}{|c|}{$\gamma=0.0$} & \multicolumn{2}{|c|}{$\gamma=1.0$} & \multicolumn{2}{|c|}{$\gamma=2.0$} \\
\hline & HAM & [26] & HAM & {$[26]$} & HAM & {$[26]$} \\
\hline 0.001 & 0.024492 & 0.02449 & 0.024831 & 0.02483 & 0.024922 & 0.02492 \\
\hline 0.003 & 0.041546 & 0.04154 & 0.042523 & 0.04252 & 0.042780 & 0.04278 \\
\hline 0.100 & 0.198031 & 0.19803 & 0.219502 & 0.21950 & 0.226096 & 0.22600 \\
\hline 0.300 & 0.303712 & 0.30371 & 0.351471 & 0.35147 & 0.366813 & 0.36681 \\
\hline 1.000 & 0.469601 & 0.46960 & 0.570475 & 0.57047 & 0.605204 & 0.60520 \\
\hline 2.000 & 0.597234 & 0.59723 & 0.743721 & 0.74372 & 0.795991 & 0.79599 \\
\hline 3.000 & 0.685967 & 0.68596 & 0.865224 & 0.86522 & 0.930362 & 0.93036 \\
\hline
\end{tabular}

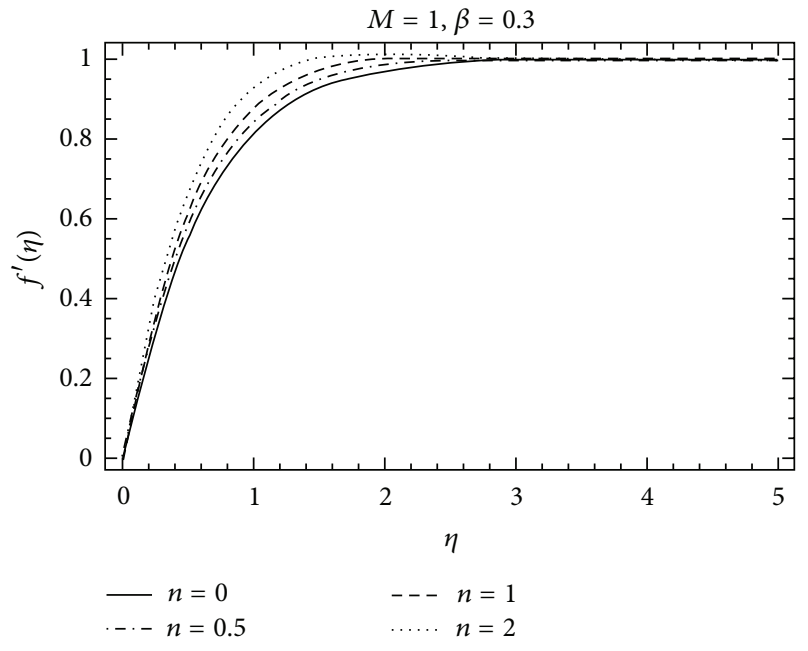

Figure 4: Influence of $n$ on $f^{\prime}$.

boundary layer thickness decreases when Pr increases. The variation of Hartman number $M$ on the temperature profile is shown in Figure 6. It is noticed that the temperature profile increases when $M$ is increased. The effects of parameter $n$ are shown in Figure 7. It is observed that an increase in the value of $n$ decreases the thermal boundary layer thickness. Figures 8 and 9 are displayed to analyze the comparison of the present analytical results with the existing numerical solutions. An

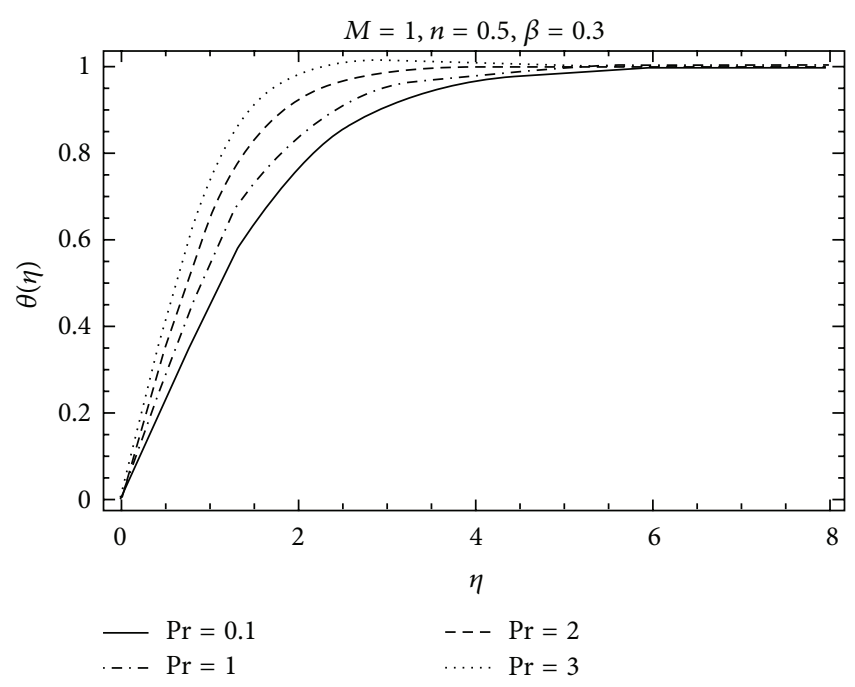

Figure 5: Influence of $\operatorname{Pr}$ on $\theta$.

excellent agreement is found between the two solutions for different values of $\gamma$. In Table 1 , we have computed the numerical values of $f(\eta), f^{\prime}(\eta)$, and $f^{\prime \prime}(\eta)$ for the comparison of the present analytical results with the numerical solution [26]. Table 2 is also a comparison between homotopy solution and numerical solution [26] in a special case. An excellent agreement is found between the two solutions. 


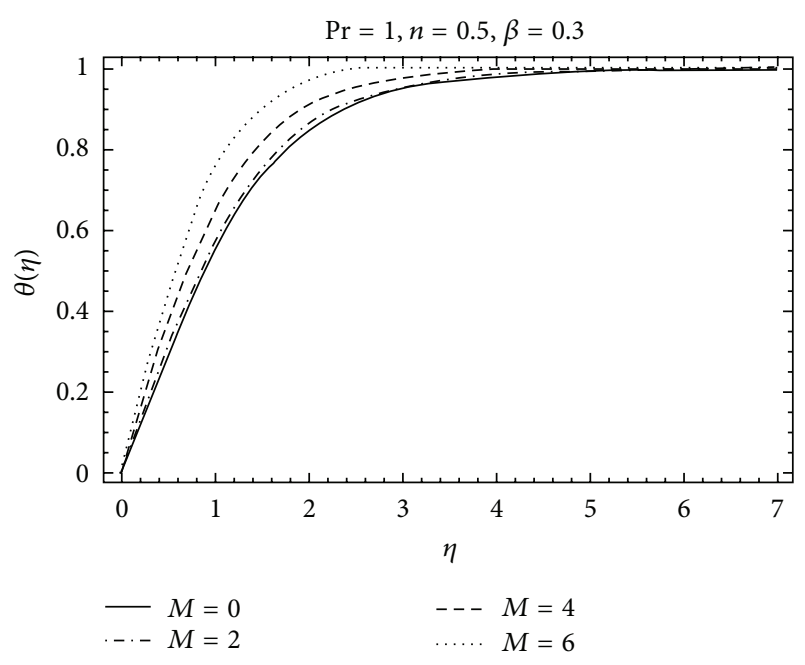

FIGURE 6: Influence of $M$ on $\theta$.

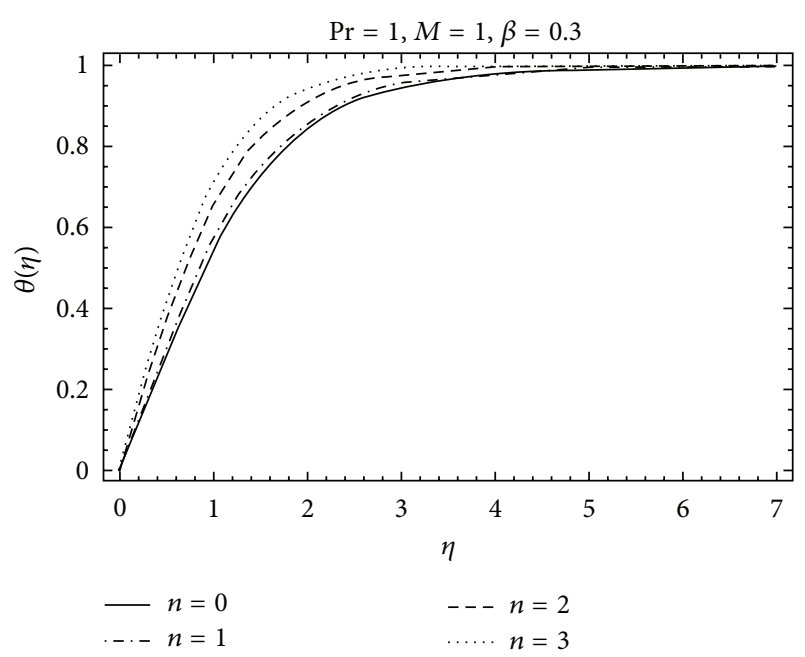

Figure 7: Influence of $n$ on $\theta$.

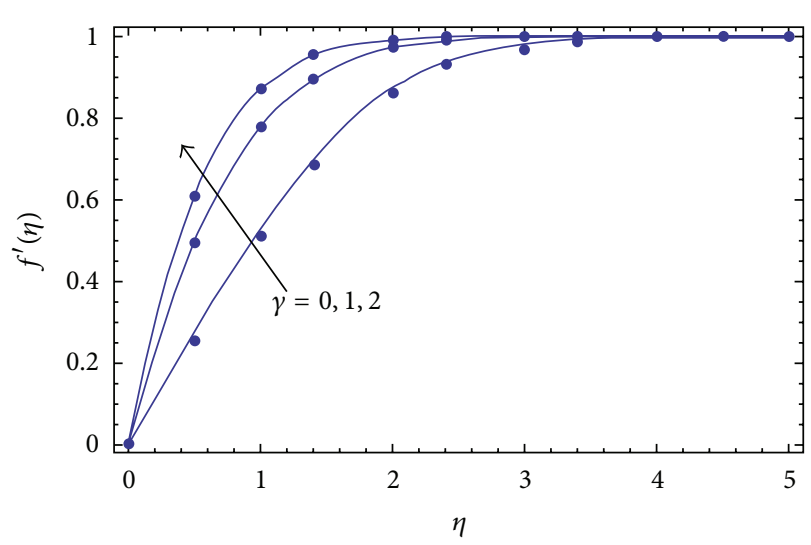

FIgURE 8: Influence of $\gamma$ on $f^{\prime}$ when $M=\beta=0$. Solid lines: HAM solution [10]; filled circles: numerical solution [26].

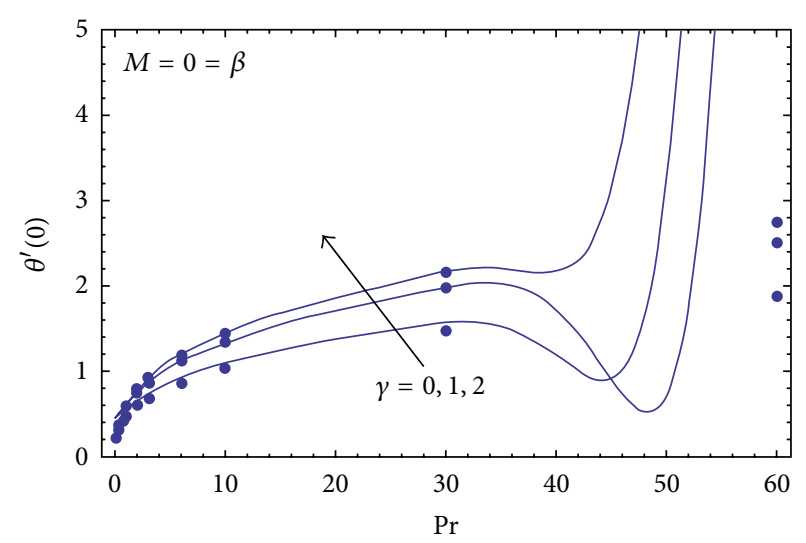

FIGURE 9: Influence of $\gamma$ on $\theta^{\prime}(0)$ when $\beta=0$. Solid lines: HAM solution [10]; filled circles: numerical solution [26].

\section{References}

[1] H. Schlichting, Boundary Layer Theory, McGraw Hill, New York, NY, USA, 6th edition, 1979.

[2] V. M. Falkner and S. W. Skan, "Some approximate solutions of the boundary layer equations," Philosophical Magazine, vol. 12, pp. 865-896, 1931.

[3] T. Cebeci and H. B. Keller, "Shooting and parallel shooting methods for solving the Falkner-Skan boundary-layer equation," Journal of Computational Physics, vol. 7, no. 2, pp. 289300, 1971.

[4] S. P. Hastings and W. Troy, "Oscillatory solutions of the FalknerSkan equation," Proceedings of the Royal Society of London A, vol. 397, no. 1813, pp. 415-418, 1985.

[5] E. F. F. Botta, F. J. Hut, and A. E. P. Veldman, "The role of periodic solutions in the Falkner-Skan problem for $\lambda>0$," Journal of Engineering Mathematics, vol. 20, no. 1, pp. 81-93, 1986.

[6] E. Alizadeh, M. Farhadi, K. Sedighi, H. R. Ebrahimi-Kebria, and A. Ghafourian, "Solution of the Falkner-Skan equation for wedge by Adomian Decomposition Method," Communications in Nonlinear Science and Numerical Simulation, vol. 14, no. 3, pp. 724-733, 2009.

[7] S. Abbasbandy and T. Hayat, "Solution of the MHD FalknerSkan flow by Hankel-Padé method," Physics Letters A, vol. 373, no. 7, pp. 731-734, 2009.

[8] S. Abbasbandy and T. Hayat, "Solution of the MHD FalknerSkan flow by homotopy analysis method," Communications in Nonlinear Science and Numerical Simulation, vol. 14, no. 9-10, pp. 3591-3598, 2009.

[9] E. Magyari, "Falkner-Skan flows past moving boundaries: an exactly solvable case," Acta Mechanica, vol. 203, no. 1-2, pp. 1321, 2009.

[10] B. Yao, "Series solution of the temperature distribution in the Falkner-Skan wedge flow by the homotopy analysis method," European Journal of Mechanics B, vol. 28, no. 5, pp. 689-693, 2009.

[11] B. Yao, "Approximate analytical solution to the Falkner-Skan wedge flow with the permeable wall of uniform suction," Communications in Nonlinear Science and Numerical Simulation, vol. 14, no. 8, pp. 3320-3326, 2009.

[12] K. R. Rajagopal, A. S. Gupta, and T. Y. Na, "A note on the falknerskan flows of a non-newtonian fluid," International Journal of Non-Linear Mechanics, vol. 18, no. 4, pp. 313-320, 1983. 
[13] M. Massoudi and M. Ramezan, "Effect of injection or suction on the Falkner-Skan flows of second grade fluids," International Journal of Non-Linear Mechanics, vol. 24, no. 3, pp. 221-227, 1989.

[14] Y. J. Kim, “The Falkner-Skan wedge flows of power-law fluids embedded in a porous medium," Transport in Porous Media, vol. 44, no. 2, pp. 267-279, 2001.

[15] D. O. Olagunju, "The Falkner-Skan flow of a viscoelastic fluid," International Journal of Non-Linear Mechanics, vol. 41, pp. 825829, 2006.

[16] S. J. Liao, Beyond Perturbation: Introduction to homotopy Analysis Method, Chapman and Hall, CRC Press, Boca Raton, Fla, USA, 2003.

[17] S. J. Liao, "Notes on the homotopy analysis method: some definitions and theorems," Communications in Nonlinear Science and Numerical Simulation, vol. 14, pp. 983-997, 2009.

[18] S.-J. Liao, "A general approach to get series solution of non-similarity boundary-layer flows," Communications in Nonlinear Science and Numerical Simulation, vol. 14, no. 5, pp. 2144-2159, 2009.

[19] S. J. Liao, "On the homotopy analysis method for nonlinear problems," Applied Mathematics and Computation, vol. 147, no. 2, pp. 499-513, 2004.

[20] T. Hayat, M. Qasim, and Z. Abbas, "Homotopy solution for the unsteady three-dimensional MHD flow and mass transfer in a porous space," Communications in Nonlinear Science and Numerical Simulation, vol. 15, no. 9, pp. 2375-2387, 2010.

[21] I. Hashim, O. Abdulaziz, and S. Momani, "The homotopy analysis method for Cauchy reaction diffusion problems," Physics Letters A, vol. 372, pp. 613-618, 2008.

[22] I. Hashim, O. Abdulaziz, and S. Momani, "Homotopy analysis method for fractional IVPs," Communications in Nonlinear Science and Numerical Simulation, vol. 14, no. 3, pp. 674-684, 2009.

[23] A. S. Bataineh, M. S. M. Noorani, and I. Hashim, "Solutions of time-dependent Emden-Fowler type equations by homotopy analysis method," Physics Letters A, vol. 371, no. 1-2, pp. 72-82, 2007.

[24] A. S. Bataineh, M. S. M. Noorani, and I. Hashim, "On a new reliable modification of homotopy analysis method," Communications in Nonlinear Science and Numerical Simulation, vol. 14, no. 2, pp. 409-423, 2009.

[25] M. Dehghan and R. Salehi, "The use of homotopy analysis method to solve the time-dependent nonlinear Eikonal partial differential equation," Zeitschrift fur Naturforschung A, vol. 66, no. 5, pp. 259-271, 2011.

[26] F. M. White, Viscous Fluid Flow, McGraw-Hill, New York, NY, USA, 2nd edition, 1991. 




Advances in

Operations Research

mansans

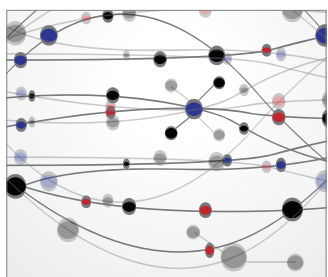

The Scientific World Journal
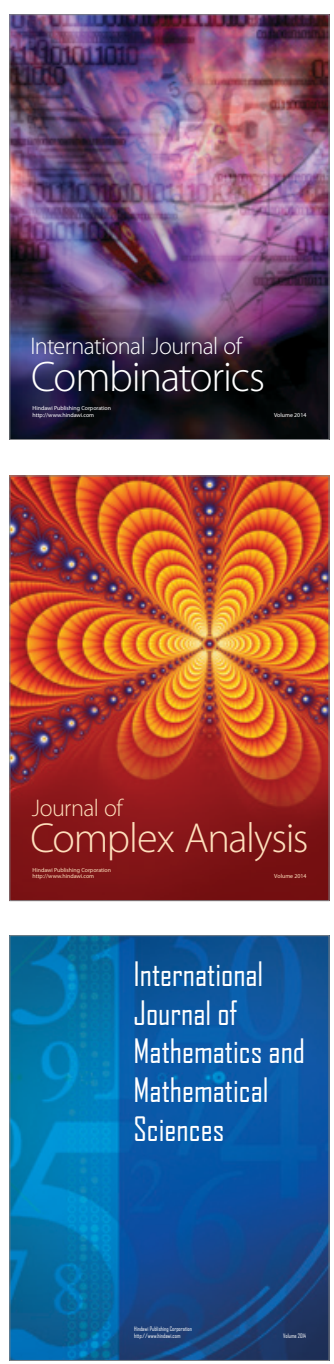
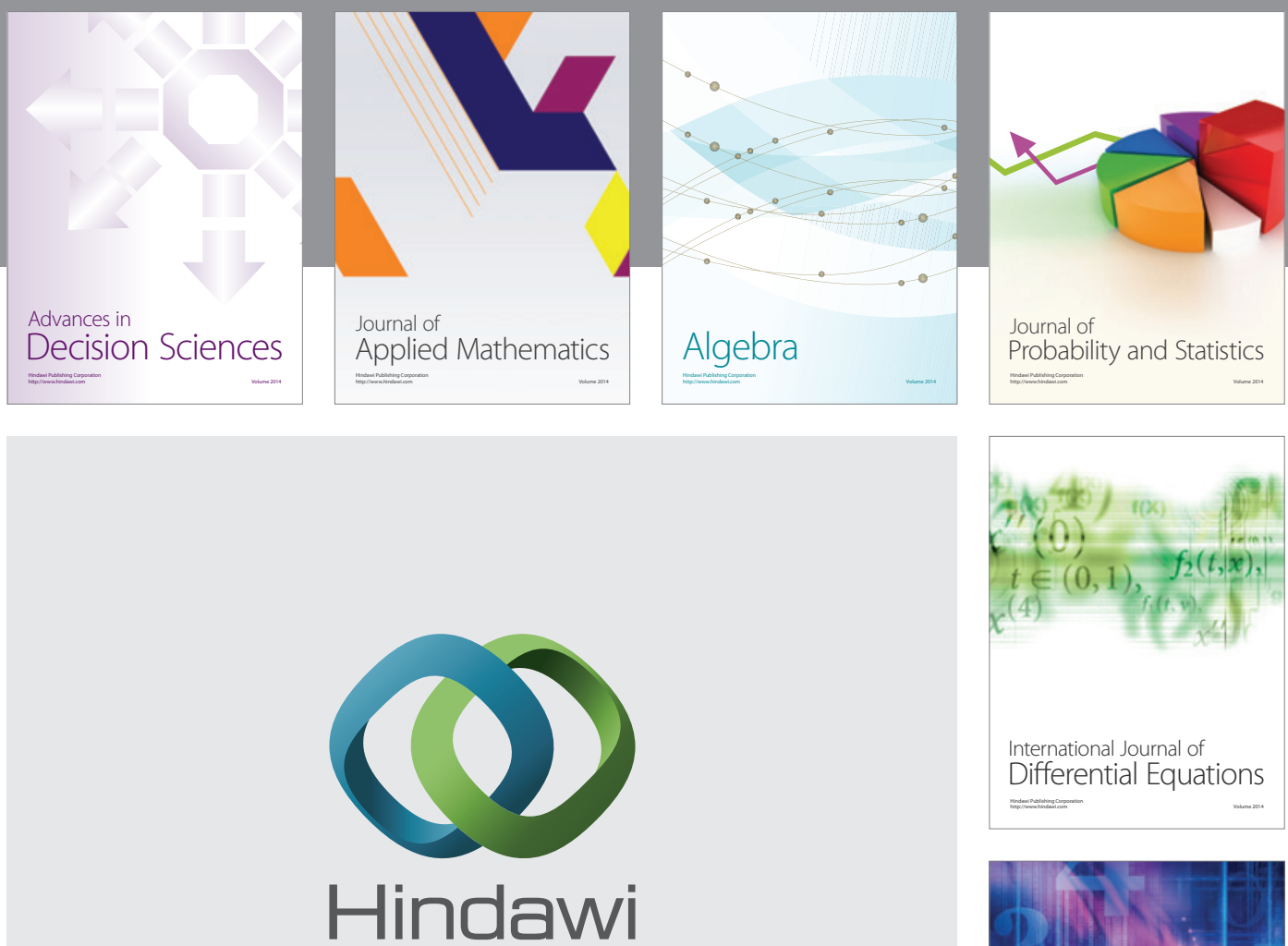

Submit your manuscripts at http://www.hindawi.com
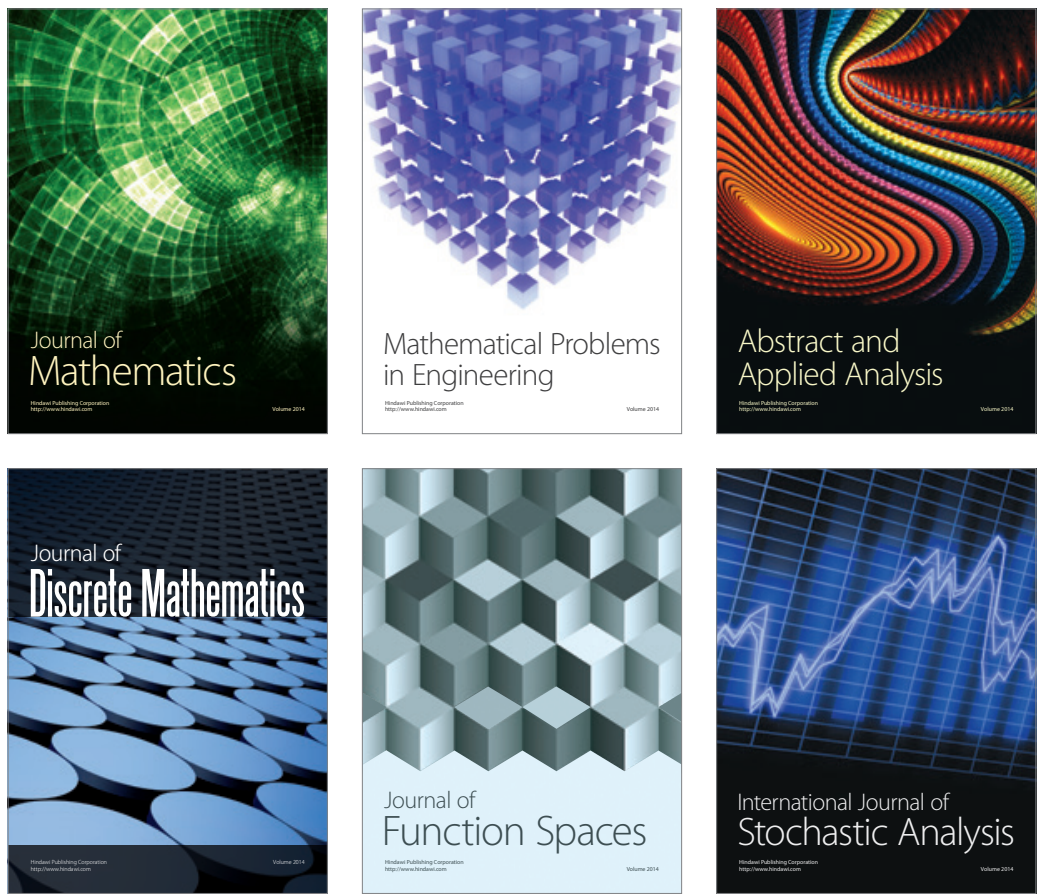

Journal of

Function Spaces



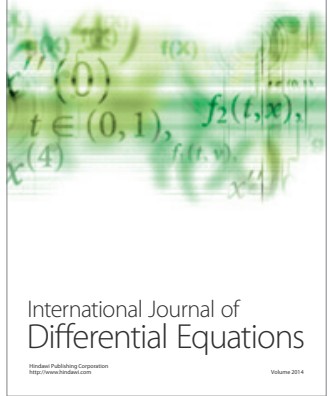
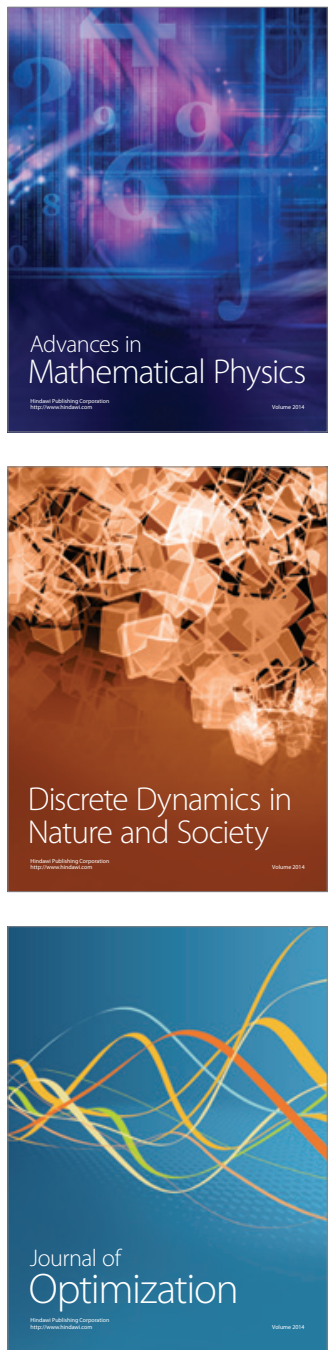